\title{
The Concept of a Device for the Redo Transcatheter Mitral Valve Implantation
}

DOI: 10.17691/stm2017.9.3.01

Received December 8, 2016

I.Y. Zhuravleva, MD, DSc, Professor, Head of Bioprosthesis Laboratory';

D.V. Nushtaev, PhD, Engineer';

T.V. Timchenko, Junior Researcher, Bioprosthesis Laboratory';

D.V. Trebushat, Senior Processing Engineer ${ }^{3}$;

A.P. Mayorov, Head of the Laboratory of Laser Medical Technologies4;

S.I. Zheleznev, MD, DSc, Professor, Chief Researcher, Centre of New Surgical Technologies';

D.P. Demidov, Physician, Cardiovascular Surgeon';

A.V. Bogachev-Prokophiev, MD, DSc, Head of Centre of New Surgical Technologies ${ }^{1}$

${ }^{1}$ Meshalkin Siberian Biomedical Research Center, 15 Rechkunovskaya St., Novosibirsk, 630055,

Russian Federation;

${ }^{2}$ TESIS, office 705, 18 Yunnatov St., Moscow, 127083, Russian Federation;

${ }^{3}$ Angioline Interventional Device, 18 Inzhenernaya St., Novosibirsk, 630090, Russian Federation;

${ }^{4}$ Institute of Laser Physics, Siberian Branch, Russian Academy of Sciences,

13/3 Academician Lavrentiev Avenue, Novosibirsk, 630090, Russian Federation

The aim of the investigation is the development of the heart valve prosthesis and transcatheter delivery system for the replacement of a failed mitral biological prosthesis.

Materials and Methods. Calculations and construction of three-dimensional models and drawings have been performed in the Computer-Aided Design environment CATIA V5 (Dassault Systèmes, France). The process of the product shaping has been simulated by the terminal element analysis in Abaqus Software (Dassault Systèmes, France). Prototypes of the stents have been made of nitinol tubes by Angioline Interventional Device company (Novosibirsk, Russia) using a laser cutting technique with outer diameter of $7.0 \mathrm{~mm}$ and wall thickness $0.5 \mathrm{~mm}$ (Vascotube $\mathrm{GmbH}$, Germany). Bioprosthetic leaflets and the cover have been cut using a laser station Melas-Cardio (Institute of Laser Physics, Siberian Branch, Russian Academy of Sciences, Novosibirsk). The valve assemblage has been hand-made using surgical sutures $8 / 0$.

Results. The self-expanding biological prosthesis design is based on the anatomy of the left heart, considering the presence of previously implanted bioprosthesis in the mitral position. The nitinol stent of cellular structure consists of a cuff, the body and the outlet part. It is round-shaped that corresponds to the shape of a standard suture bioprostheses. One-third of the cuff adjacent to the mitralaortic contact area forms a 70-degree angle to the mitral annulus plane. In the outlet part there are three hook retainers of the previously implanted bioprosthetic leaflets. For prosthesis repositioning during implantation there are two-way (on the cuff and on hook retainers) retainers and complementary latches in the central catheter of the delivery system, they providing the connection of the valve with the delivery system in both transapical and transatrial access. The valve is provided with radiopaque markers to facilitate the positioning. Valve leaflets and the cover have been made of porcine pericardium treated with epoxy compound. The cover coats the inner surface of the stent cuff and a part of the body.

Conclusion. We developed the prototype of a device for the redo transcatheter mitral valve implantation. Further preclinical testing is necessary to evaluate a safety of the medical device.

Key words: transcatheter valve implantation; bioprosthesis; mitral valve; "valve-to-valve" technique.

For contacts: Irina Y. Zhuravleva, e-mail: juravl_irina@mail.ru 
Transcatheter heart valve replacement is a breakthrough technology in modern cardiology and minimally invasive cardiac surgery since it enables to perform valve surgeries in those patients who were previously considered to be inoperable. Nowadays, over 150 thousand transcatheter aortic valve implantations (TAVI) have been performed worldwide and the number keeps increasing on average by $40 \%$ annularly [1]. In recent years, transcatheter mitral valve implantation (TMVI) related to the development of such type bioprostheses is growing dramatically. More than 10-year TMVI development delaying from TAVI is due to complex mitral valve anatomy [2-4]. Currently, the main efforts are aimed to design bioprostheses and delivery systems for their implantation in a native mitral valve. Therefore, their construction is based on anatomical and functional features of the mitral valve: nonplanarity of mitral valve ring, the presence of two leaflets, complex subvalvular apparatus, etc. [5]. All of them are self-expanding bioprostheses, which anchor due to a nitinol stent releasing into the left heart chambers and attaching to two leaflets of the mitral valve [2]. Presently, balloon expandable valves with a low profile and a simple cylindrical form, which initially designed for aortic position, are frequently used in previously implanted failed bioprostheses with a rounded section, a rigid frame and three axisymmetric leaflets [6]. There are limited data in the literature concerning the constructions designed for transcatheter implantation in a position of a failed mitral bioprosthesis.

The aim of the investigation was to develop the heart valve prosthesis and a transcatheter delivery system to replace a failed mitral biological prosthesis.

Materials and Methods. While developing a new model we took into consideration the following theoretical prerequisites and medico-technical requirements:

1) a valve should be self-expandable, since the effect of a balloon opening in a failed calcified bioprosthesis can lead to arterial embolism;

2) a valve should have three anchors in the left ventricle, according to the number of bioprosthesis leaflets, because they are the only fixation zone of the ventricular part for a transcatheter valve;

3) a valve is to be packed in a catheter, which is no more than $8 \mathrm{~mm}(24 \mathrm{Fr})$ in diameter, i.e. the diameter of the nitinol tube used for valve's stent producing is to be no more than $7 \mathrm{~mm}$;

4) a delivery system should be unified for transapical and transatrial approach, i.e. if the structure is unified it provides primary release of either a ventricular or an atrial part of the valve;

5 ) the delivery system length should be convenient for minimally invasive procedures, i.e. it should not exceed $50 \mathrm{~cm}$;

6) to prevent dislocation into the left ventricle, a valve should have a cuff enabling to avoid injuries in a thin zone of an aorto-mitral contact, on the one hand, and avoid malposition of the zone towards the left ventricle, on the other hand;

7) to ease the positioning during the implantation a valve should be equipped with radiopaque markers.

Medical nitinol (corresponding to ASTM F-2063 standard) alloy (Vascotube $\mathrm{GmbH}$, Germany) exhibiting superelasticity due to austenite-martensite transition occurring in high mechanical stress was chosen as a stent material.

The design and construction of three-dimensional stent models, as well as the main drafts and drawings were performed in the computer-aided design environment CATIA V5 (Dassault Systèmes, France).

In order to check the construction consistency and capability to make a finishing diameter during the production cycle, we used the finite element analysis to form up the product. Initial stent models corresponding to the samples obtained in a laser cutting phase were imported to the engineering analysis Abaqus (Dassault Systèmes, France) for grid generation consisting of 19,200 cubic elements followed by opening modeling up to the desired diameters: $23,25,27,29$ and $31 \mathrm{~mm}$ using a cylindrical membrane model, which is in contact with the outer surface of the mesh regardless of traction. Radial symmetry restrictions for central connector nodes $\left(U_{2}=0\right.$ in a cylindrical coordinate system) were imposed on a model. The parameters of superelastic titanium nickelide, Auricchio model, were chosen as the material properties [7]: the stress of an early transformation phase of austenite-martensite $(\mathrm{A}-\mathrm{M}) \sigma_{L}{ }^{S}=460 \mathrm{MPa}$; the stress of the end transformation phase $A-M \sigma_{L}{ }^{E}=540 \mathrm{MPa}$; the stress of an early transformation phase of martensiteaustenite $(\mathrm{M}-\mathrm{A}) \sigma_{U}{ }^{S}=370 \mathrm{MPa}$; the stress of the end transformation phase $\mathrm{M}-\mathrm{A} \sigma_{U}{ }^{E}=220 \mathrm{MPa}$; martensite modulus of elasticity $E_{M}=27,000 \mathrm{MPa}$; austenite modulus of elasticity $E_{A}=45,000 \mathrm{MPa}$; stress transformation range $\varepsilon^{L}=0.05$.

To reveal the critical stress nodes in a construction, we used Mises yield criterion - a maximal value in finiteelement mesh nodes. Literature data: 1,070 $\mathrm{MPa}$ - was used as a threshold value for this parameter [8].

Experimental sample models of stents were manufactured by Angioline International Device (Novosibirsk, Russia) using a laser cutting technique, on A1 Laser International (USA) with electrochemical polishing from a tube, $7.0 \mathrm{~mm}$ in an external diameter and wall thickness of $0.5 \mathrm{~mm}$. Shape training in a salt bath was performed to assign a cylindrical form and form up a finishing diameter. The temperature control of phase transformation of stents was maintained using a differential scanning calorimeter DSC-3 (Mettler Toledo, USA).

When developing curve-drawing pattern to cut out a leaflet valve apparatus, we used the approaches developed before for designing leaflet apparatus of aortic valves $[9,10]$. The patterns were transformed into a corresponding program CNC of a laser hardware system Melas-Cardio (Institute of Laser Physics, 
Siberian Branch, Russian Academy of Sciences, Novosibirsk) designed for biological material cutting. Leaflet apparatus laser cut from porcine pericardium sheets, with the ethylene alcohol diglycidyl ether treatment, was manually assembled on a stent using a surgical technique, suture material and instruments. Internal biological lining of the stent body and cuffs were made from xenopericardium as well.

Results and Discussion. The first transcatheter valve implantation in a failed mitral bioprosthesis (valve-invalve technology) was performed in 2005 by Boudjemline et al. [11]. Balloon expandable valves known in pediatric cardiosurgery (NuMed Inc., USA) were put in five sheep after the conventional suture implantation of a Mosaic bioprosthesis (Medtronic, USA) by direct transcatheter approach via the left atrium. Two years later, Walther et al. [12] experimentally tested a transapical approach to a mitral bioprosthesis, as well as proved the capability of implantation of transcatheter aortal valve bioprosthesis Sapien (Edwards Lifesciences, USA).

First clinical results "valve-in-valve" implantation was published in 2010 by Webb et al. [13]. Later in 2010, a multicenter study - the Valve-in-Valve International Data was initiated [14] and devoted to the use "valvein-valve" and "valve-in-ring" for all intracardiac positions. By the year 2016 the first results in 347 patients with mitral bioprosthesis dysfunction were found [15]. Malposition of transcatheter valves was revealed in $6.6 \%$ cases, the exit pathway obstruction of the left ventricle - in $2.6 \%$ cases, residual regurgitation - in $2.6 \%$ cases; 30 -day lethality being $7.7 \%$.

It should be noted that a transcatheter valve Sapien was used in $90 \%$ patients enrolled in the study, the rest patients had Inovare (Braile Biomédica, Brazil) or Melody (Medtronic, USA) valves [16]. All these bioprostheses are balloon expandable; Sapien and Inovare are initially designed for transcatheter replacement of a native aortal valve, while Melody is used in dysfunctional right valve-containing conduits. There are several reasons for choosing these prostheses. Firstly, an anatomical reason: these are the most low-profile bioprostheses, cylindrically shaped, with three leaflets; the rest bioprostheses for TAVI have a higher profile and to a greater extent are structurally associated with the aortal valve and the ascending aorta anatomy [15]. Secondly, a clinical testing of first bioprostheses for TMVI was held only in 2014, and currently, the number of recipients worldwide using these valves has hardly reached 100 [17]. Thirdly, TMVI bioprostheses are designed so that to take into consideration anatomical features of mitral valvular apparatus, and do not suitable to be fixed in a previously implanted bioprosthesis [2].

The design of a bioprosthesis we have developed (Figure 1) takes into consideration both: the anatomy of the left heart chambers and the presence of the previously implanted bioprosthesis in the mitral position.

A cuff and a stent of the prosthesis have round shape corresponding to the shape of standard suture bioprostheses designed for mitral position implantation. In addition, two-thirds of the cuff circumference are located in the mitral annulus plane (i.e. in the cuff plane of the previously implanted bioprosthesis), while the one-third is adjacent to a mitral-aortal contact zone, and is directed at $70^{\circ}$ angle to this plane. On the one hand, it reduces the cuff traumatism, and on the other hand prevents the cuff zone from displacement towards the left ventricle due to the cuff contact with the previously implanted bioprosthesis. A similar approach was used in a mitral transcatheter bioprosthesis Tiara (Neovasc Inc., USA) [18], however, a part of the cuff in the prosthesis adjacent to the mitral-aortal contact zone is situated at the right angle to the mitral ring plane that can result in bioprosthesis malposition.

In the outlet part there are three hook retainers that are corresponding to the number of the previously implanted bioprosthetic leaflets. The retainers have a curve directed to the inflow zone of the stent frame, i.e. to the direction opposite to transprosthetic blood flow. Such geometry enables both: fix a transcatheter bioprosthesis on the left ventricular side, and also squeeze and immobilize the leaflets of the previously implanted bioprosthesis. The similar design of retainers was

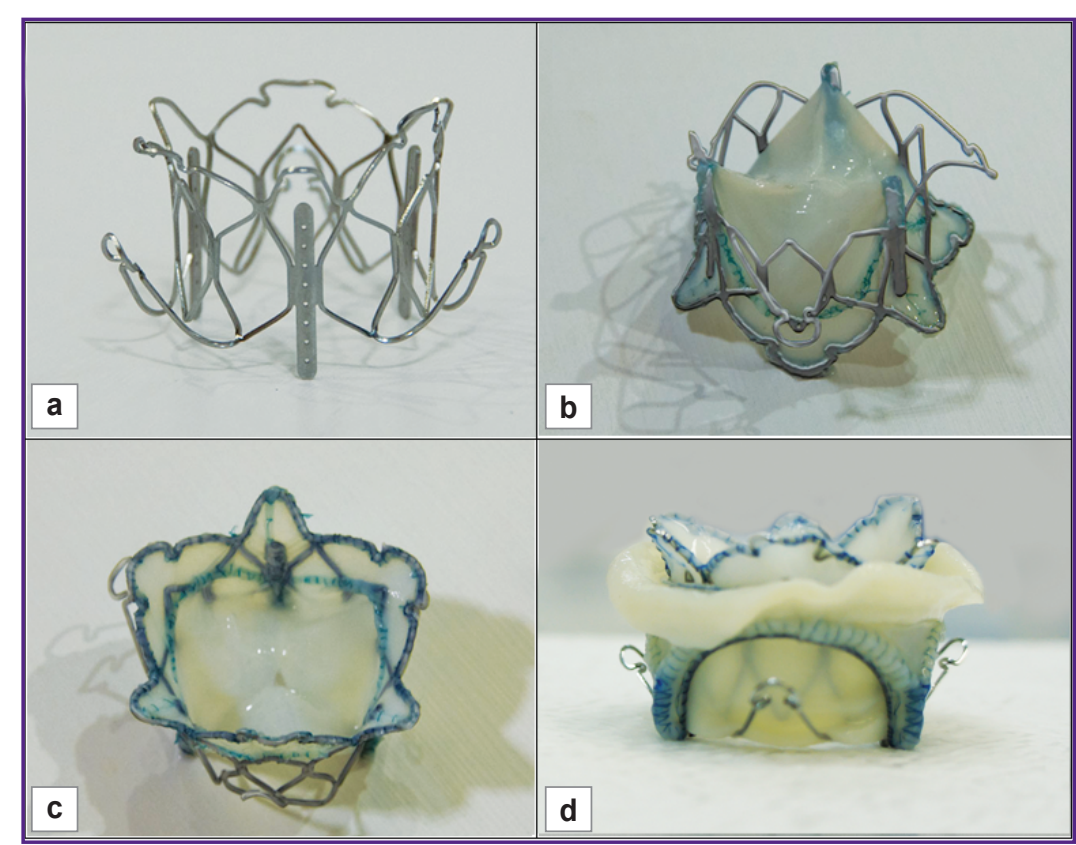

Figure 1. The appearance of the developed valve model:

(a) nitinol stent; (b) the valve assembled, the output side; (c) the valve, inflow side; (d) the developed model implanted in UniLine bioprosthesis (NeoCor, Kemerovo, Russia) 
used in a transcatheter bioprosthesis Fortis (Edwards Lifesciences, USA) [19], however, there are only two of them in this prosthesis, and they are contralateral to the stent frame, since they are designed for the anterior and posterior leaflets of the native mitral valve.

The designed bioprosthesis also differs from Tiara and Fortis valves in the fact that to provide prosthesis repositioning in implantation, it has two-way (on the cuff and on hook retainers) retainers and complementary latches in the central catheter of the delivery system (Figure 2 (a)). It provides the valve connection with a delivery system before the moment of ultimate positioning, which is possible after a bioprosthesis is attached in one of two anatomical areas: in the atrium (in a transapical approach) (Figure 2 (b)) or in the ventricle (in a transatrial approach) (Figure 2 (c)). A bioprosthesis could be completely released from a delivery system only after ultimate positioning.

A bioprosthesis frame is a cylindrical structure consisting of cells joined together and connecting three vertical supports. Nitinol properties allow the frame to anchor in a rigid frame of the previously implanted



Figure 2. The valve position in a system:

(a) transapical approach, 1 - retainers of a stent on complementary latches of the central catheter; (b) transapical approach, bioprosthesis positioning in the left atrium, the valve is connected with the system; (c) transatrial approach, the bioprosthesis is positioned in the left ventricle, the valve is connected with the system bioprosthesis due to radial forces. The supports serve to form commissural cores of leaflets, and are located symmetrically with respect to the central prosthetic axis at a $120^{\circ}$ angle from one another that corresponds to the location of supports in most standard suture bioprosthetic models. The supports of a transcatheter bioprosthesis in implantation should be positioned by the supports of the previously implanted bioprosthesis (Figure 2 (d)). The valve is provided with radiopaque markers to facilitate the positioning. A marker located at the upper point of the middle of the cuff, adjacent to the mitral-aortal contact zone, in transapical approach should be situated at the center between the supports of the previously implanted bioprosthesis, the point being in the outlet part of the left ventricle. In transapical approach, the markers located in border points of three hook retainers should be directed to the centre of the distance between the supports of the previously implanted bioprosthesis.

It should be noted, that in positioning transcatheter valves, a radio-opacity problem of a replaceable bioprosthesis arises. Among various bioprostheses in worldwide market, only two have radiopaque markers: Perimount (Edwards Lifesciences, USA) and Hancock II (Medtronic, USA). For the rest models it is recommended to use a preliminary expanded balloon in order to state a valve location [15]. Among bioprostheses used in Russia, only bioprosthesis UniLine (NeoCor, Kemerovo, Russia) has a fully radiopaque nitinol contour.

The designed frame model is technologically feasible. Moreover, the design approach applied enable to unify a manufacturing process at a laser cutting stage of a nitinol tube: all end typical sizes of a product — from 23 to $31 \mathrm{~mm}$ - were as a part of full design. The differences of the frame diameters resulted from shape training (Figure 3). The computer modeling of the shape training process showed that the highest stress areas were placed into the bottom of the hook retainers, however, absolute values did not exceed the threshold value strength limit (1,070 MPa): 664.4 and 790.6 MPa for 23 (the smallest) and $31 \mathrm{~mm}$ (the largest) in diameter, respectively.

Differential scanning calorimetry found the shift of phase transfer of nitinol stent of a bioprosthesis due to shape training: the temperature of the finishing phase transfer to austenite $A_{f}$ determining functional characteristics of a product was $28.35^{\circ} \mathrm{C}$ (Figure 4). Thus, the structure of the supporting stent is a transient state austenite-martensite, i.e. hysteresis plateau. The approach is typical for designing most self-expanding structures due to stable mechanical properties - in relation to constant radial forces of the supporting stent.

The bioprosthesis coat covers completely the cuff inside and just partially spreads the frame corresponding to the stent of the previously implanted bioprosthesis (Figure 1 (b), (c)). Uncoated stent cells create no obstacle to blood flow including that in the outlet part of the left ventricle. In order to enhance hemocompatibility, they can be titanium oxynitride coated [20]. Moreover, 


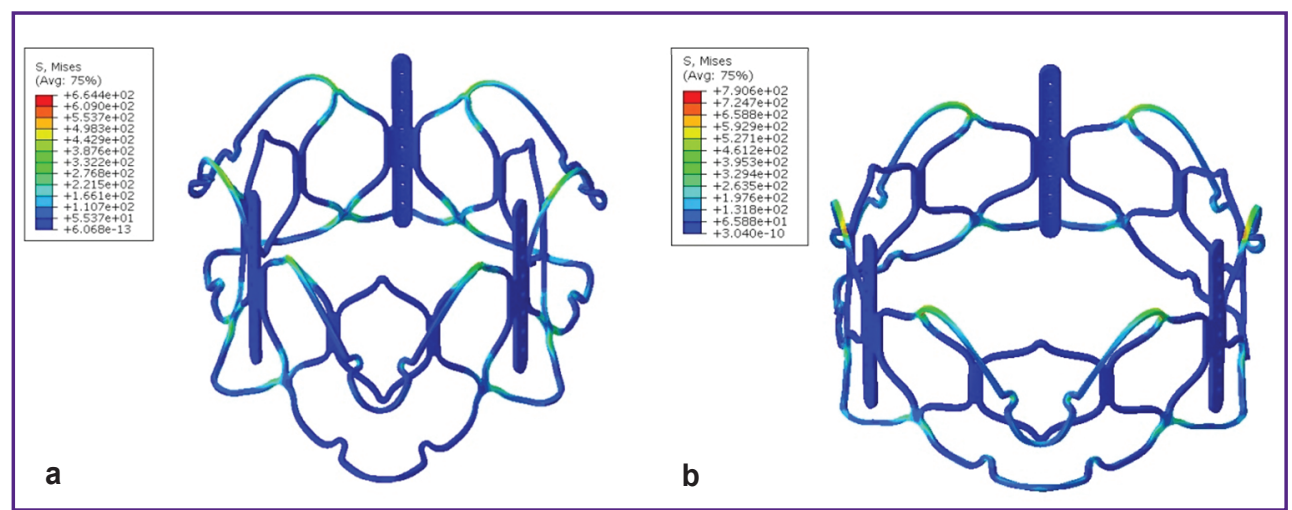

Figure 3. Mises voltage distribution in cellular nodes of stents, in an open state, diameter $23 \mathrm{~mm}$ (a) and $31 \mathrm{~mm}$ (b)

covering material minimization is determined by the necessity to be packed in a delivery system of a small diameter - not more than $8 \mathrm{~mm}$ (24 Fr). The same reason underlies the choice of porcine pericardium as a material for a leaflet apparatus. Porcine pericardium is known to be thinner and durable than bovine pericardium [10], therefore its usage is preferable in those bioprosthetic constructions, which are designed to be packed in a catheter system of minor diameter.

Catheter systems of minor diameter are designed for percutaneous approach with

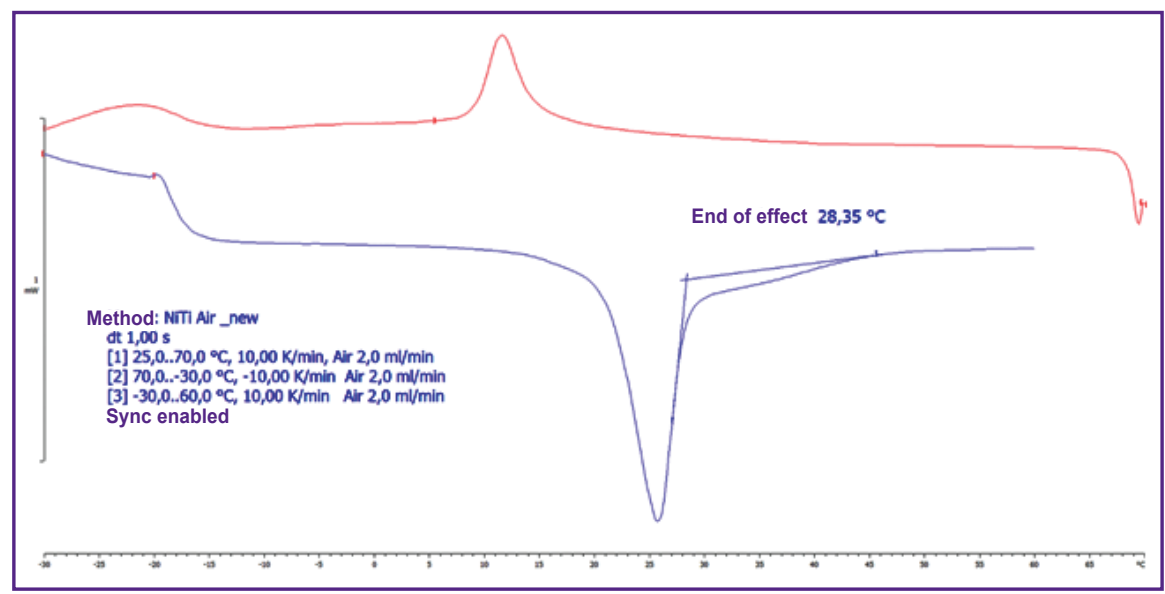

Figure 4. Differential scanning calorimetry findings of a sample of nitinol state after thermal forming catheterization via femoral or jugular vein into the right atrium followed by puncture in the left atrium. Then it goes through the mitral valve, a bioprosthesis is being released from a delivery system. After positioning, the atrial part of a bioprosthesis is released [6, 15, 21]. It should be noted that when replacing a previously implanted bioprosthesis using Sapien valves in a delivery system of 14-16 Fr (4.7$5.3 \mathrm{~mm}$ ), transvessel approach is used increasingly frequently: in $2014-2016$ - in $25.4 \%$ cases, while in 2013 - just in 15\% cases [14]. However, currently, most prostheses designed for TMVI are packed in the systems with diameters of over $30 \mathrm{Fr}[2,22]$ that makes transvessel access to the mitral valve unfeasible, and determines the choice in favor of a mini-thoracotomic approach: transapical or transatrial suggested by Bruschi et al. in 2012 [23].

We also consider it to be possible to use thoracoscopic transatrial access to a mitral bioprosthesis. There are no data of TMVI applying using totally thoracoscopy approach however, the approach is quite compatible with percutaneous. It enables to perform the procedure even in patients with marked post-operative adhesions

in thorax, since if it is impossible to deliver the system via the left atrium, a catheter with a valve can "enter" the right atrium followed by transseptal puncture of the left atrium. This is precisely why a transcatheter bioprosthesis model presented in this survey is designed to be packed in a system not more than $8 \mathrm{~mm}(24 \mathrm{Fr}$ ) in diameter: a prosthesis should pass freely through a manipulation port of a thoracoscope, 12-13 $\mathrm{mm}$ in diameter.

Conclusion. We developed a technologically reproducible model for transcatheter replacement of a degenerated mitral bioprosthesis previously implanted. Further preclinical testing including bench tests as well as orthotopic implantation in large laboratory animals are necessary to evaluate its workability as a medical device.

Study Funding. The work was funded by Russian Science Foundation grant (16-15-10315).

Conflicts of Interest. The authors declare of possible conflicts of interest with Research Institute for Complex Issues of Cardiovascular Diseases (Kemerovo) consisting in scientific competition in the sphere of development of implants for cardiovascular surgery. 


\section{References}

1. Cribier A., Durand E., Eltchaninoff H. Patient selection for TAVI in 2014: is it justified to treat low- or intermediaterisk patients? The cardiologist's view. Eurolntervention 2014; 10(U): U16-U21, https://doi.org/10.4244/eijv10sua3.

2. Preston-Maher G.L., Torii R., Burriesci G. A technical review of minimally invasive mitral valve replacements Cardiovasc Eng Technol 2015; 6(2): 174-184, https://doi. org/10.1007/s13239-014-0203-9.

3. Dolgov V.Y., Ovcharenko E.A., Klyshnikov K.Y., Sizova I.N., Kudryavtseva Y.A., Barbarash L.S. Automated method to analyze geometry and topology of mitral valve fibrous ring. Sovremennye tehnologii v medicine 2016; 8(2): 22-30, https://doi.org/10.17691/stm2016.8.2.03.

4. Young M., Erdemir A., Stucke S., Klatte R., Davis B., Navia J.L. Simulation based design and evaluation of a transcatheter mitral heart valve frame. J Med Device 2012; 6(3): 031005, https://doi.org/10.1115/1.4007182.

5. Zamorano J.L., González-Gómez A., Lancellotti P. Mitral valve anatomy: implications for transcatheter mitral valve interventions. Eurolntervention 2014; 10(U): U106-U111, https://doi.org/10.4244/eijv10sua15.

6. Gallo M., Dvir D., Demertzis S., Pedrazzini G., Berdajs D., Ferrari E. Transcatheter valve-in-valve implantation for degenerated bioprosthetic aortic and mitral valves. Expert Rev Med Devices 2016; 13(8): 749-758, https://doi.org/10.108 0/17434440.2016.1207521.

7. Auricchio F., Taylor R.L. Shape-memory alloys: modelling and numerical simulations of the finite-strain superelastic behavior. Comput Methods Appl Mech Eng 1997; 143(1-2): 175-194, https://doi.org/10.1016/s0045-7825(96)01147-4.

8. Ovcharenko E.A., Klyshnikov K.U., Yuzhalin A.E., Savrasov G.V., Kokov A.N., Batranin A.V., Ganyukov V.I., Kudryavtseva Y.A. Modeling of transcatheter aortic valve replacement: patient specific vs general approaches based on finite element analysis. Comput Biol Med 2016; 69: 29-36, https://doi.org/10.1016/j.compbiomed.2015.12.001.

9. Ovcharenko E.A., Klyshnikov K.Y., Nushtaev D.V., Savrasov G.V., Barbarash L.S. Investigation of the tubular leaflet geometry of an aortic heart valve prosthesis by finiteelement analysis. Biophysics 2015; 60(5): 827-834, https://doi. org/10.1134/s0006350915050152.

10. Ovcharenko E.A., Klyshnikov K.Y., Glushkova T.V., Nyshtaev D.V., Kudryavtseva Y.A., Savrasov G.V. Xenopericardial graft selection for valve apparatus of transcatheter heart valve bioprosthesis. Biomed Eng 2016; 49(5): 253-257, https://doi.org/10.1007/s10527-016-9543-0.

11. Boudjemline $Y$. New insights in minimally invasive valve replacement: description of a cooperative approach for the offpump replacement of mitral valves. Eur Heart J 2005; 26(19): 2013-2017, https://doi.org/10.1093/eurheartj/ehi307.

12. Walther T., Falk V., Dewey T., Kempfert J., Emrich F., Pfannmüller B., Bröske P., Borger M.A., Schuler G., Mack M., Mohr F.W. Valve-in-a-valve concept for transcatheter minimally invasive repeat xenograft implantation. J Am Coll Cardiol 2007; 50(1): 56-60, https://doi.org/10.1016/j.jacc.2007.03.030.
13. Webb J.G., Wood D.A., Ye J., Gurvitch R., Masson J.B., Rodés-Cabau J., Osten M., Horlick E., Wendler O., Dumont E., Carere R.G., Wijesinghe N., Nietlispach F., Johnson M., Thompson C.R., Moss R., Leipsic J., Munt B., Lichtenstein S.V., Cheung A. Transcatheter valve-in-valve implantation for failed bioprosthetic heart valves. Circulation 2010; 121(16): 18481857, https://doi.org/10.1161/circulationaha.109.924613.

14. Simonato M., Webb J., Kornowski R., Vahanian A., Frerker C., Nissen H., Bleiziffer S., Duncan A., RodésCabau J., Attizzani G.F., Horlick E., Latib A., Bekeredjian R., Barbanti M., Lefevre T., Cerillo A., Hernández J.M., Bruschi G., Spargias K., ladanza A., Brecker S., Palma J.H., Finkelstein A., Abdel-Wahab M., Lemos P., Petronio A.S., Champagnac D., Sinning J.M., Salizzoni S., Napodano M., Fiorina C., Marzocchi A., Leon M., Dvir D. Transcatheter replacement of failed bioprosthetic valves: large multicenter assessment of the effect of implantation depth on hemodynamics after aortic valve-in-valve. Circ Cardiovasc Interv 2016; 9(6): e003651, https://doi.org/10.1161/circinterventions.115.003651.

15. Paradis J.-M., Del Trigo M., Puri R., Rodés-Cabau J. Transcatheter valve-in-valve and valve-in-ring for treating aortic and mitral surgical prosthetic dysfunction. J Am Coll Cardiol 2015; 66(18): 2019-2037, https://doi.org/10.1016/j. jacc.2015.09.015.

16. Dvir D., Webb J. Mitral valve-in-valve and valve-in-ring: technical aspects and procedural outcomes. Eurolntervention 2016; 12(Y): Y93-Y96, https://doi.org/10.4244/eijv12sya25.

17. Baumgarten H., Squiers J.J., Arsalan M., John M., Dimaio M.J. Defining the clinical need and indications: who are the right patients for transcatheter mitral valve replacement. J Cardiovasc Surg (Torino) 2016; 57(3): 352-359.

18. Lane R.M., Nyuli C.A. Transcatheter mitral valve prosthesis. US patent 8,579,964. 2013.

19. Chau M., Patterson M., Yi S., Geist S., Oba T. Prosthetic valve for replacing mitral valve. US patent 8,449,599. 2013.

20. Pichugin V.F., Pustovalova A.A., Konishchev M.E., Khlusov I.A., Ivanova N.M., Zhilei S., Gutor S.S. In-vitro dissolution and structural and electrokinetic characteristics of titanium-oxynitride coatings formed via reactive magnetron sputtering. J Synch Investig 2016; 10(2): 282-291, https://doi. org/10.1134/s1027451016020166.

21. Coylewright M., Cabalka A.K., Malouf J.A., Geske J.B., Pollak P.M., Suri R.M., Rihal C.S. Percutaneous mitral valve replacement using a transvenous, transseptal approach. JACC Cardiovasc Interv 2015; 8(6): 850-857, https://doi. org/10.1016/j.jcin.2015.01.028.

22. Ramlawi B., Gammie J.S. Mitral valve surgery: current minimally invasive and transcatheter options. Methodist Debakey Cardiovasc J 2016; 12(1): 20-26, https://doi. org/10.14797/mdcj-12-1-20.

23. Bruschi G., Barosi A., Colombo P., Botta L., Oreglia J., De Marco F., Paino R., Klugmann S., Martinelli L. Direct transatrial transcatheter SAPIEN valve implantation through right minithoracotomy in a degenerated mitral bioprosthetic valve. Ann Thorac Surg 2012; 93(5): 1708-1710, https://doi. org/10.1016/j.athoracsur.2011.08.084. 